\title{
SAFETY ASSESSMENT OF SHOES FIT FOR DRIVING
}

\section{EVALUAREA SIGURANTEI ÎNCĂLTĂMINTEI POTRIVITE PENTRU ŞOFAT}

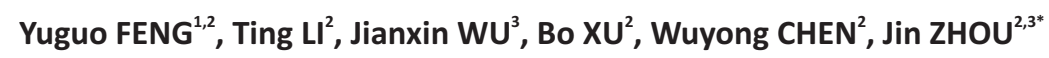 \\ ${ }^{1}$ College of Applied Technology of Xihua University, Sichuan Province, Chengdu 610065, P. R. China
}

${ }^{2}$ Key Laboratory of Leather Chemistry and Engineering of Ministry of Education, Sichuan University, Sichuan Province, Chengdu 610065, P. R. China

${ }^{3}$ Science Lab, Zhejiang Red Dragonfly Footwear Co., LTD., Zhejiang Province, Wenzhou 325100, P. R. China

\begin{abstract}
SAFETY ASSESSMENT OF SHOES FIT FOR DRIVING
ABSTRACT. This study was aimed to explore a suitable heel height of shoes for driving. Fourteen healthy female students were recruited and five pairs of footwear with varied heel heights were randomly arranged to each participant; their insole plantar pressure and ankle motion in plantar and dorsal direction whilst stepping on the power and break pedal were recorded in a Suzuki 1.3L AT car, where the driving position was on the left side. One-mask model was used and peak pressure (PP) (Kpa), contact area (CA) $\left(\mathrm{cm}^{2}\right)$ and pressure time integral (PTI) (s.Kpa) under forefoot area were calculated. Our results show that in terms of ankle motion, it increased with the relative heel height; in terms of insole pressure, only the CA was sensitive to the augment of relative heel height. Based on the significant differences, $30 \mathrm{~mm}$ heel height was a criterion; when the heel height exceeded $30 \mathrm{~mm}$, the ankle motion range and CA were significantly increased. Overall, the safe heel-height of driving shoe should not exceed $30 \mathrm{~mm}$.
\end{abstract}

KEY WORDS: driving; safety; insole pressure; ankle motion; high-heeled footwear

\section{EVALUAREA SIGURANTEI ÎNCALTAMINTEI POTRIVITE PENTRU SOFAT}

REZUMAT. Acest studiu a avut ca scop gasirea unor pantofi cu toc de înaltime adecvata pentru sofat. S-au recrutat paisprezece studente sanatoase si li s-au distribuit aleatoriu cinci perechi de încaltaminte cu înaltimi de toc variate; s-au înregistrat presiunea plantara la nivelul brantului si amplitudinea articulara în directie plantara si dorsala în timpul apasarii pe pedalele de acceleratie si de frâna într-o masina Suzuki model 1.3L AT, cu locul soferului pe partea stânga. S-a utilizat modelul cu o singura zona de analiza si s-au calculat presiunea maxima (PP) (Kpa), zona de contact (CA) ( $\left.\mathrm{cm}^{2}\right)$ si integrala presiune-timp (PTI) (s·Kpa) în zona antepiciorului. Rezultatele noastre arata ca, în ceea ce priveste amplitudinea miscarii gleznei, aceasta a crescut odata cu înaltimea relativa a tocului; în ceea ce priveste presiunea la nivelul brantului, doar CA a fost sensibila la cresterea înaltimii relative a tocului. Pe baza diferentelor semnificative, a reiesit ca tocul cu înaltime de $30 \mathrm{~mm}$ a fost cel adecvat; când înaltimea tocului a depasit $30 \mathrm{~mm}$, amplitudinea articulara si CA au crescut semnificativ. În general, pentru siguranta, înaltimea tocului încaltamintei potrivite pentru sofat nu trebuie sa depaseasca $30 \mathrm{~mm}$.

CUVINTE CHEIE: sofat; siguranta; presiune la nivelul brantului; amplitudine articulara; încaltaminte cu toc înalt

\section{ÉVALUATION DE LA SÉCURITÉ DES CHAUSSURES ADAPTÉES À LA CONDUITE}

RÉSUMÉ. Cette étude visait à explorer une hauteur de talon de chaussures appropriée pour la conduite. Quatorze étudiants femmes en bonne santé ont été recrutées et cinq paires de chaussures avec des hauteurs de talons variées ont été disposées au hasard à chacun des participants; on a enregistré leur pression plantaire dans la région de la semelle et le mouvement de la cheville dans les directions plantaire et dorsale en appuyant sur l'accélérateur et le frein dans une voiture Suzuki 1.3L AT, où la position de conduite est sur le côté gauche. Le modèle à une région a été utilisé et on a calculé la pression maximale (PP) (Kpa), la zone de contact (CA) $\left(\mathrm{cm}^{2}\right)$ et l'intégrale pression-temps (PTI) (s·Kpa) dans la zone de l'avant-pied. Nos résultats montrent que le mouvement de la cheville a augmenté avec la hauteur relative de talon; en termes de pression de semelle, seulement la CA a été sensible à l'augment de la hauteur relative de talon. Sur la base des différences significatives, la hauteur de talon de $30 \mathrm{~mm}$ a été appropriée; lorsque la hauteur du talon a dépassé $30 \mathrm{~mm}$, le mouvement de la cheville et la CA ont augmenté significativement. Dans l'ensemble, pour la sécurité, la hauteur de talon des chaussures de conduite ne doit pas dépasser $30 \mathrm{~mm}$.

MOTSCLES: conduite; sécurité; pression dans la région de la semelle; mouvement de la cheville; chaussures à talon haut

\section{INTRODUCTION}

The Global Status Report on Road Safety 2013 presented that worldwide the total number of road traffic deaths remains unacceptably high at 1.24 million per year [1]. With the rapid economic development of China, the volume of motor vehicle increased dramatically in the past 30 years, which led to the

\section{INTRODUCERE}

Raportul Global pe anul 2013 privind siguranţa rutieră a precizat că numărul total la nivel mondial al deceselor datorate traficului rutier rămâne inacceptabil de ridicat la 1,24 milioane pe an [1]. Odată cu dezvoltarea economică rapidă a Chinei, numărul de autovehicule a crescut dramatic în ultimii 30 de ani,

* Correspondence to: Jin ZHOU, Key Laboratory of Leather Chemistry and engineering of Ministry of Education, Sichuan Univ., Sichuan Province, Chengdu 610065, Science Lab, Zhejiang red dragonfly footwear Co., LTD., Zhejiang Province, Wenzhou 325100, zj_scu@qq.com 
sharply increasing road traffic accidents [2]. According to an official report [3], China topped the list in terms of number of traffic deaths and traffic accidents in the world. As the main participators of the road traffic system, drivers play a dominant role in traffic accidents, being responsible for more than $90 \%$ of the road traffic accidents; meanwhile, $30 \%$ of all the accidents were triggered by female drivers $[4,5]$. Further, a survey disclosed that $51.4 \%$ of the family car users were female drivers $[6,7]$, thereby, their habitude of driving with heeled shoes, particularly high-heeled shoes became a potential risk factor of driving safety.

Current studies focusing on the analysis of risk factor in driving, such as psychological performance [8], drivers' view and behavior [2]; whereas, how the heel-heighted footwear affected with the driving, especially in the action of power and break were not scientifically considered in the current database. There are several studies on footwear design with varying heel height [9-11]. This study was aimed to explore a suitable heel height for driving by comparing the insole plantar pressure and ankle motion range of five types of footwear with varied heel heights.

\section{METHODS}

\section{Subjects}

In this study, 14 healthy female students were recruited. The inclusion criteria are shown below: (1) foot length range: $230 \pm 2.5 \mathrm{~mm}$; (2) without foot deformity and foot surgery history in the past three months; (3) body mass index (BMI) range: 15 22; (4) qualified with driving license; (5) at least one year driving experience. The demographic information of those subjects is shown below: the mean age is 21 23, the mean height is $151 \sim 159 \mathrm{~cm}$, the mean weight is $44.7^{\sim} 54.9 \mathrm{~kg}$, and the mean BMl is 19.19 22.23. All the procedures were informed to the participants and their formal approval was obtained before the trial begun. The processes of this study were strictly following the principles of Helsinki declaration.

\section{Footwear}

Five pairs of shoes were selected for trials (Figure 1). Test shoe No. 1 (S1) was professional ceea ce a condus la creşterea bruscă a accidentelor rutiere [2]. Potrivit unui raport oficial [3], China se află în fruntea listei în ceea ce priveşte numărul de decese şi accidente datorate traficului rutier. Ca participanţi principali la sistemul de trafic rutier, şoferii joacă un rol dominant în accidentele rutiere, fiind responsabili de mai mult de $90 \%$ din accidentele rutiere, în timp ce 30\% din numărul total de accidente au fost declanşate de şoferi de sex feminin $[4,5]$. Mai mult, un studiu a dezvăluit faptul că 51,4\% din şoferii de maşini de familie sunt femei $[6,7]$; astfel, obiceiul acestora de a conduce purtând pantofi cu toc, şi mai ales pantofi cu toc înalt, a devenit un potenţial factor de risc privind siguranţa în conducere.

Studiile actuale se axează pe analiza factorilor de risc în şofat, cum ar fi performanţa psihologică [8], vederea şi comportamentul şoferilor [2]; în timp ce modul în care încălţămintea cu toc înalt afectează şofatul, în special la acţionarea pedalelor de acceleraţie şi de frână, nu este luat în considerare din punct de vedere ştiinţific. Există mai multe studii referitoare la proiectarea încălţămintei cu înălţimea tocului diferită [9-11]. Acest studiu are scopul de a stabili o înălţime a tocului adecvată pentru şofat prin compararea presiunii plantare la nivelul branţului şi amplitudinea articulară utilizând cinci tipuri de încălţăminte cuînălţimi de toc variate.

\section{METODE}

\section{Subiecţi}

În cadrul acestui studiu s-au recrutat 14 studente sănătoase. Criteriile de incluziune sunt prezentate în continuare: (1) lungimea labei piciorului: $230 \pm 2,5 \mathrm{~mm}$; (2), inexistenţa deformărilor sau intervenţiilor chirurgicale la nivelul piciorului în ultimele trei luni; (3) interval indice de masă corporală (IMC): 15 22; (4) deţinerea unui permis de conducere; (5) experienţă în şofat de cel puţin un an. Informaţiile demografice ale subieç̧ilor sunt prezentate mai jos: vârsta medie de 21 23 de ani, înălţimea medie de 151 159 cm, greutatea medie de 44,7 54,9 kg, iar IMC mediu de 19.19 22.23. Participantele au fost informate cu privire la toate procedurile şi s-a obţinut aprobarea oficială de la acestea înainte de începerea studiului. Procesele din cadrul acestui studiu au urmatîn modstrict principiile din Declaraţia dela Helsinki.

\section{Încălţăminte}

S-au ales cinci perechi de pantofi pentru studiu (Figura 1). Perechea nr. 1 (S1) a fost o pereche de pantofi 
driving shoe from a relevant brand; test shoe No. 2 was sports shoe (S2), while shoe No. 3 (S3) was lowheeled nude shoe; shoe No. 4 (S4) was mid-heeled nude shoe and shoe No. 5 (S5) is wedge sandals. The heel height of those five pairs of test shoes were showed in Table 1. profesionişti de marcă pentru şofat; perechea nr. 2 a fost o pereche de pantofi sport (S2), iar perechea $\mathrm{nr} .3$ (S3) a fost o pereche de pantofi cu toc mic; perechea nr. 4 (S4) a fost o pereche de pantofi cu toc mediu, iar perechea nr. 5 (S5) a fost o pereche de sandale cu platformă. Înălţimea tocului celor cinci perechi de pantofi testaţi este redată în Tabelul 1.

Table 1: The heel height of shoes for trials

Tabelul 1: Înălţimea tocului pantofilor testaţi

\begin{tabular}{|c|c|c|c|}
$\begin{array}{c}\text { No. } \\
\text { Nr. } \\
\text { crt. }\end{array}$ & $\begin{array}{c}\text { Foot spring }(\mathrm{mm}) \\
\text { Ridicarea } \\
\text { piciorului }(\mathrm{mm})\end{array}$ & $\begin{array}{c}\text { Relative heel height }(\mathrm{mm}) \\
\text { Inălţimea relativă } a \\
\text { tocului }(\mathrm{mm})\end{array}$ & $\begin{array}{c}\text { Absolute heel height }(\mathrm{mm}) \\
\text { Inălţimea absolută a tocului } \\
(\mathrm{mm})\end{array}$ \\
\hline \hline S1 & 7 & 10 & 3 \\
\hline S2 & 15 & 29 & 14 \\
\hline S3 & 7 & 34 & 30 \\
\hline S4 & 4 & 73 & 69 \\
\hline S5 & 33 & 100 & 67 \\
\hline
\end{tabular}

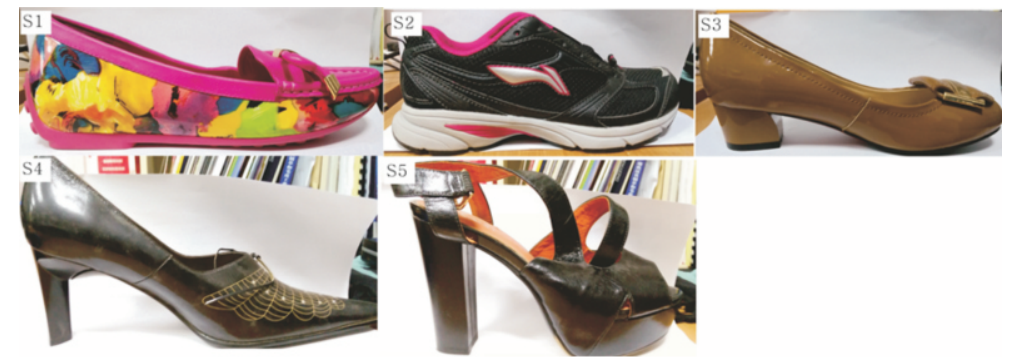

Figure 1. Test shoes $\mathrm{S} 1$ to $\mathrm{S} 5$

Figura 1. Pantofii de testare, de la S1 la S5

\section{Data Collection}

The test was simulated in a real car without driving on the road. The car was Suzuki 1.3L AT, the driving position was on the left side. At first, a randomized shoes code was arranged to each participant and two minutes warm up was provided; moreover, their insole plantar pressure whilst stepping on the power and break pedal with normal force was recorded by Pedar-X system (Pedar-X, Novel Inc., Germany). One-mask model (Figure 2) was used and only forefoot of insole pressure was recorded. The forefoot region was defined as the area between $41 \%$ to $80 \%$ foot length. Variables of peak pressure (PP) (Kpa), contact area (CA) $\left(\mathrm{cm}^{2}\right)$ and pressure time integral (PTI) (s.Kpa) were calculated. Their ankle motion range (MR) $\left({ }^{\circ}\right)$ of plantar flexion and dorsal flexion was obtained by a digital goniometer (DA-100, Kailibo, China), where the

\section{Colectarea datelor}

Testul a fost simulat într-o maşină reală, fără a conduce pe şosea. Maşina a fost Suzuki model 1.3L AT, cu locul şoferului pe partea stângă. La început, participantelor li s-a repartizat o pereche de pantofi la întâmplare şi s-au alocat două minute pentru acomodare. Apoi presiunea plantară la nivelul branţului în timpul apăsării pedalelor de acceleraţie şi de frână cu putere normală a fost înregistrată utilizând sistemul Pedar-X (Pedar-X, Novel Inc., Germania). S-a utilizat modelul cu o singură zonă (Figura 2), înregistrându-se presiunea doar în zona antepiciorului. Zona antepiciorului a fost definită ca zona situată între 41\% şi $80 \%$ din lungimea totală a piciorului. S-au calculat variabilele presiune maximă (PP) (Kpa), zona de contact (CA) $\left(\mathrm{cm}^{2}\right)$ şi integrala presiune-timp (PTI) (s.Kpa). Amplitudinea articulară (MR) $\left(^{\circ}\right)$ la flexia plantară şi cea dorsală a fost obţinută cu ajutorul unui goniometru digital 
three key points for angle measurement were set at medial lower tibial, the first metatarsal head and the swell of the medial heel. The range of MR was defined as the stepping on the pedal in the neutral position and in the position could not be stepped any more. At least three successful measurements were required.

\section{Data Processing and Statistical Analysis}

Three trials' data were first averaged for each variable and results of One-sample KolmogorovSmirnov test showed that all the data were following with the normal distribution. Comparisons within the varied footwear in terms of insole pressure and ankle motion range were evaluated by Paired-t test from SPSS (V16.0, SPSS Inc. USA) with the significant difference of 0.05 and confidence interval of $95 \%$.
(DA-100, Kailibo, China), stabilindu-se cele trei punctecheie de măsurare a unghiului în zona medie inferioară a tibiei, la primul metatarsian şi la proeminenţa călcâiului. Intervalul amplitudinii articulare a fost definit ca intervalul de la poziţionarea piciorului pe pedală în poziţie neutră şi până la apăsarea acesteia până la capăt. Au fost necesare cel puţin trei măsurători viabile.

\section{Prelucrarea datelor şi analiza statistică}

S-a făcut media a trei încercări pentru fiecare variabilă, iar rezultatele testului Kolmogorov-Smirnov pentru un singur eşantion au arătat că toate datele au o distribuţie normală. Comparaţiile între diferitele tipuri de încălţăminte din punctul de vedere al presiunii plantare la nivelul branţului şi al amplitudinii articulare au fost evaluate prin testul t pereche efectuat cu SPSS (v16.0, SPSS Inc. SUA), cu diferenţa semnificativă de 0,05 şi intervalul de încredere de 95\%.

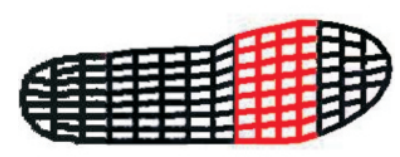

Figure 2. One-mask model of insole pressure measure

Figura 2. Modelul cu o singură zonă pentru măsurarea presiunii la nivelul branţului

\section{RESULTS AND DISCUSSION}

Angle Parameters

\section{REZULTATE ŞI DISCUTII}

\section{Parametrii unghiului}

Table 2: Foot angle in driving position when wearing test shoes

Tabelul 2: Unghiul piciorului la purtarea pantofilor de testare în poziţia de şofat

\begin{tabular}{|c|c|c|c|c|c|}
\hline $\begin{array}{l}\text { Action } \\
\text { Acţiune }\end{array}$ & $\mathrm{N}$ & $\begin{array}{l}\text { Shoes } \\
\text { Pantofi }\end{array}$ & $\begin{array}{c}\text { MR } \\
\text { Mean }\left({ }^{\circ}\right) \\
\text { Amplitudinea } \\
\text { medie a mişcării }\left({ }^{\circ}\right)\end{array}$ & $\begin{array}{c}\mathrm{MR} \\
\mathrm{SD} \\
\text { Abatere standard }\end{array}$ & Sig. \\
\hline \multirow{5}{*}{$\begin{array}{l}\text { Brake } \\
\text { Frână }\end{array}$} & \multirow{5}{*}{14} & S1 & 146.4 & 10.1 & \multirow{5}{*}{$\mathrm{S} 1<\mathrm{S} 4=0.004, \mathrm{~S} 2<\mathrm{S} 4=0.004, \mathrm{~S} 3<\mathrm{S} 4=0.048$} \\
\hline & & S2 & 146.4 & 9.7 & \\
\hline & & S3 & 148.7 & 8.0 & \\
\hline & & S4 & 153.7 & 8.1 & \\
\hline & & S5 & 151.1 & 10.3 & \\
\hline \multirow{5}{*}{$\begin{array}{c}\text { Power } \\
\text { Acceleraţie }\end{array}$} & \multirow{5}{*}{14} & S1 & 150.2 & 8.1 & \multirow{5}{*}{$\begin{array}{c}\mathrm{S} 1<\mathrm{S} 4=0.001, \mathrm{~S} 1<\mathrm{S} 5=0.003 \\
\mathrm{~S} 2<\mathrm{S} 4=0.001, \mathrm{~S} 2<\mathrm{S} 5=0.002, \mathrm{~S} 3<\mathrm{S} 4=0.050\end{array}$} \\
\hline & & S2 & 149.9 & 10.1 & \\
\hline & & S3 & 153.6 & 8.1 & \\
\hline & & S4 & 158.3 & 8.1 & \\
\hline & & S5 & 157.3 & 9.9 & \\
\hline
\end{tabular}




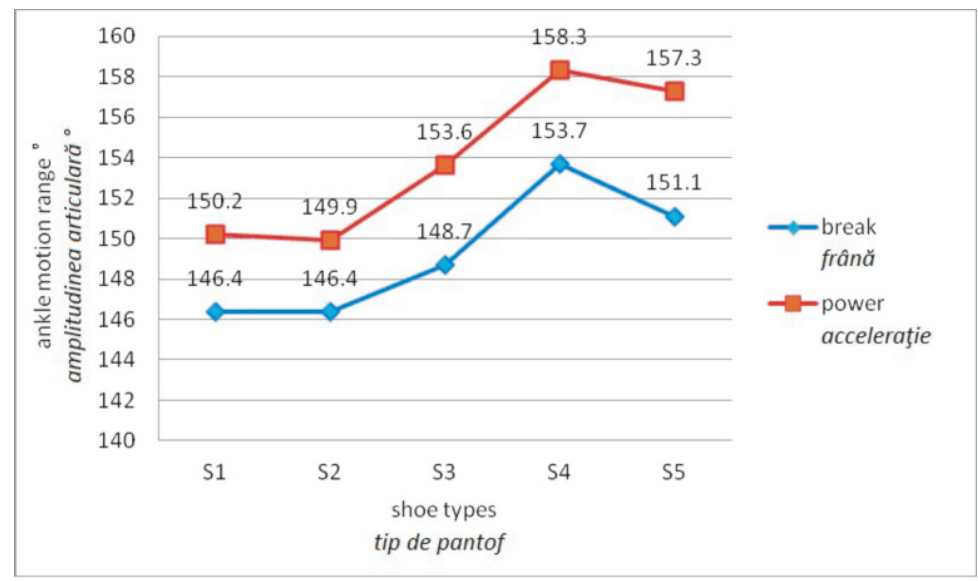

Figure 3. Foot angle in driving position when wearing test shoes

Figura 3. Unghiul piciorului la purtarea pantofilor de testare în poziţia de şofat

From Table 2 and Figure 3, S4 shows a significantly larger movement angles than S1, S2 and S3 in both brake or power condition $(\mathrm{S} 1<\mathrm{S} 4=0.004, \mathrm{~S} 2<\mathrm{S} 4=$ $0.004, \mathrm{~S} 3<\mathrm{S} 4=0.048$ ). In addition, the movement angle of S5 is significantly larger than $\mathrm{S} 2$ in the brake condition $(\mathrm{S} 1<\mathrm{S} 4=0.001, \mathrm{~S} 1<\mathrm{S} 5=0.003, \mathrm{~S} 2<\mathrm{S} 4=$ $0.001, \mathrm{~S} 2<\mathrm{S} 5=0.002, \mathrm{~S} 3<\mathrm{S} 4=0.050)$.

\section{The Distribution of Insole Pressure}

Comparisons within five pairs of test shoes (Table 3 and Figure 4) depicts that there is no significant difference with peak pressure and impulse in the condition of brake or power. While, in the respect of contact area, S1 is significantly less than S2 in the condition of brake $(p=0.048), S 2<S 3(p=0.022), S 2<$ S4 $(p=0.046)$.
Tabelul 2 şi Figura 3 arată că S4 prezintă o amplitudine articulară semnificativ mai mare decât $\mathrm{S} 1$, S2 şi S3, atât la frână, cât şi la acceleraţie (S1 < S4 = $0,004, \mathrm{~S} 2<\mathrm{S} 4=0,004, \mathrm{~S} 3<\mathrm{S} 4=0,048)$. În plus, amplitudinea articulară a $\mathrm{S} 5$ este semnificativ mai mare decât cea a S2 la frână $(\mathrm{S} 1<\mathrm{S} 4=0,001, \mathrm{~S} 1<\mathrm{S} 5=0,003$, $\mathrm{S} 2<\mathrm{S} 4=0,001, \mathrm{~S} 2<\mathrm{S} 5=0,002, \mathrm{~S} 3<\mathrm{S} 4=0,050)$.

\section{Distribuţia presiunii la nivelul branţului}

Comparaţiile între cele cinci perechi de pantofi de testare (Tabelul 3 şi Figura 4) arată că nu există nicio diferenţă semnificativă între presiunea maximă şi impuls la acţionarea acceleraţiei şi a frânei. În timp ce, în ceea ce priveşte zona de contact, valoarea pentru S1 este semnificativ mai mică decât cea pentru S2 la frână $(p=0,048), S 2<S 3(p=0,022), S 2<S 4(p=0,046)$.

Table 3: Insole plantar pressure distribution whilst driving

Tabelul 3: Distribuţia presiunii plantare la nivelul branţului în timpul şofatului

\begin{tabular}{|c|c|c|c|c|c|c|}
\hline $\begin{array}{l}\text { Action } \\
\text { Acţiune }\end{array}$ & $\begin{array}{l}\text { Variables } \\
\text { Variabile }\end{array}$ & $\begin{array}{l}\text { Shoes } \\
\text { Pantofi }\end{array}$ & $N$ & $\begin{array}{l}\text { Mean } \\
\text { Medie }\end{array}$ & $\begin{array}{l}\text { SD } \\
\text { Abatere } \\
\text { standard }\end{array}$ & Sig \\
\hline \multirow{10}{*}{$\begin{array}{c}\text { Power } \\
\text { Acceleraţie }\end{array}$} & \multirow{5}{*}{$\begin{array}{c}\mathrm{PP} \\
\text { (Кpa) }\end{array}$} & S1 & 12 & 68.6 & 55.5 & \multirow{5}{*}{$\begin{array}{c}\mathrm{S} 1>\mathrm{S} 2=0.605 ; \mathrm{S} 1<\mathrm{S} 3=0.220 ; \mathrm{S} 1<\mathrm{S} 4=0.175 ; \\
\mathrm{S} 1<\mathrm{S} 5=0.344 ; \mathrm{S} 2<\mathrm{S} 3=0.184 ; \mathrm{S} 2<\mathrm{S} 4=0.088 ; \\
\mathrm{S} 2<\mathrm{S} 5=0.239 ; \mathrm{S} 3<\mathrm{S} 4=0.478 ; \mathrm{S} 3>\mathrm{S} 5=0.569 ; \\
\mathrm{S} 4>\mathrm{S} 5=0.291\end{array}$} \\
\hline & & S2 & 12 & 59.2 & 37.5 & \\
\hline & & S3 & 12 & 87.1 & 47.3 & \\
\hline & & S4 & 12 & 104.1 & 63.7 & \\
\hline & & S5 & 12 & 77.8 & 48.6 & \\
\hline & \multirow{5}{*}{$\begin{array}{c}\mathrm{CA} \\
\left(\mathrm{Cm}^{2}\right)\end{array}$} & S1 & 12 & 18.8 & 10.7 & \multirow{5}{*}{$\begin{array}{c}\mathrm{S} 1>\mathrm{S} 2=0.227 ; \mathrm{S} 1<\mathrm{S} 3=0.272 ; \mathrm{S} 1<\mathrm{S} 4=0.416 ; \\
\mathrm{S} 1>\mathrm{S} 5=0.276 ; \mathrm{S} 2<\mathrm{S} 3=0.078 \\
\mathrm{~S} 2<\mathrm{S} 4=0.046 * ; \mathrm{S} 2<\mathrm{S} 5=0.825 \\
\mathrm{~S} 3<\mathrm{S} 4=0.918 ; \mathrm{S} 3>\mathrm{S} 5=0.117 ; \mathrm{S} 4>\mathrm{S} 5=0.099\end{array}$} \\
\hline & & S2 & 12 & 15.1 & 8.5 & \\
\hline & & S3 & 12 & 21.2 & 11.2 & \\
\hline & & S4 & 12 & 21.4 & 10.1 & \\
\hline & & S5 & 12 & 15.5 & 6.1 & \\
\hline
\end{tabular}


Table 3: Continued

Tabelul 3: Continuare

\begin{tabular}{|c|c|c|c|c|c|c|}
\hline $\begin{array}{l}\text { Action } \\
\text { Acţiune }\end{array}$ & $\begin{array}{l}\text { Variables } \\
\text { Variabile }\end{array}$ & $\begin{array}{l}\text { Shoes } \\
\text { Pantofi }\end{array}$ & $\mathrm{N}$ & $\begin{array}{l}\text { Mean } \\
\text { Medie }\end{array}$ & $\begin{array}{l}\text { SD } \\
\text { Abatere } \\
\text { standard }\end{array}$ & Sig \\
\hline \multirow{5}{*}{$\begin{array}{c}\text { Power } \\
\text { Acceleraţie }\end{array}$} & \multirow{5}{*}{$\begin{array}{c}\text { PTI } \\
\text { (s Kpa) }\end{array}$} & S1 & 12 & 39.0 & 38.4 & \multirow{5}{*}{$\begin{array}{c}\mathrm{S} 1<\mathrm{S} 2=0.779 ; \mathrm{S} 1<\mathrm{S} 3=0.499 ; \mathrm{S} 1<\mathrm{S} 4=0.416 ; \\
\mathrm{S} 1<\mathrm{S} 5=0.528 ; \mathrm{S} 2<\mathrm{S} 3=0.895 ; \mathrm{S} 2<\mathrm{S} 4=0.230 \\
\mathrm{~S} 2<\mathrm{S} 5=0.829 ; \mathrm{S} 3<\mathrm{S} 4=0.156 ; \mathrm{S} 3<\mathrm{S} 5=0.876 ; \\
\mathrm{S} 4>\mathrm{S} 5=0.195\end{array}$} \\
\hline & & S2 & 12 & 43.6 & 41.0 & \\
\hline & & S3 & 12 & 45.5 & 27.6 & \\
\hline & & S4 & 12 & 76.3 & 73.4 & \\
\hline & & S5 & 12 & 47.1 & 37.3 & \\
\hline \multirow{15}{*}{$\begin{array}{l}\text { Brake } \\
\text { Frână }\end{array}$} & \multirow{5}{*}{$\begin{array}{l}\text { PP } \\
\text { (Kpa) }\end{array}$} & S1 & 12 & 42.0 & 23.9 & \multirow{5}{*}{$\begin{array}{c}\mathrm{S} 1>\mathrm{S} 2=0.508 ; \mathrm{S} 1<\mathrm{S} 3=0.530 ; \mathrm{S} 1<\mathrm{S} 4=0.543 ; \\
\mathrm{S} 1>\mathrm{S} 5=0.965 ; \mathrm{S} 2<\mathrm{S} 3=0.317 ; \mathrm{S} 2<\mathrm{S} 4=0.377 \\
\mathrm{~S} 2<\mathrm{S} 5=0.537 ; \mathrm{S} 3<\mathrm{S} 4=0.967 ; \mathrm{S} 3>\mathrm{S} 5=0.588 \\
\mathrm{~S} 4>\mathrm{S} 5=0.405\end{array}$} \\
\hline & & S2 & 12 & 36.5 & 20.7 & \\
\hline & & S3 & 12 & 46.9 & 24.9 & \\
\hline & & S4 & 12 & 47.4 & 29.6 & \\
\hline & & S5 & 12 & 41.7 & 18.3 & \\
\hline & \multirow{5}{*}{$\begin{array}{c}\mathrm{CA} \\
\left(\mathrm{Cm}^{2}\right)\end{array}$} & S1 & 12 & 10.4 & 4.0 & \multirow{5}{*}{$\begin{aligned} & \mathrm{S} 1>\mathrm{S} 2= 0.048^{*} ; \mathrm{S} 1<\mathrm{S} 3=0.537 ; \\
& \mathrm{S} 1<\mathrm{S} 4=0.859 ; \mathrm{S} 1>\mathrm{S} 5=0.882 ; \\
& \mathrm{S} 2<\mathrm{S} 3=0.022^{*} ; \mathrm{S} 2<\mathrm{S} 4=0.032^{*} ; \\
& \mathrm{S} 2<\mathrm{S} 5=0.060 ; \mathrm{S} 3>\mathrm{S} 4=0.895 ; \mathrm{S} 3>\mathrm{S} 5=0.644 ; \\
& \mathrm{S} 4>\mathrm{S} 5=0.791\end{aligned}$} \\
\hline & & S2 & 12 & 7.1 & 3.0 & \\
\hline & & S3 & 12 & 10.9 & 3.5 & \\
\hline & & S4 & 12 & 10.7 & 5.3 & \\
\hline & & S5 & 12 & 10.1 & 4.3 & \\
\hline & \multirow{5}{*}{$\begin{array}{c}\text { PTI } \\
\text { (s Kpa) }\end{array}$} & S1 & 12 & 16.9 & 13.0 & \multirow{5}{*}{$\begin{array}{rl}\mathrm{S} 1<\mathrm{S} 2=0.215 ; & \mathrm{S} 1<\mathrm{S} 3=0.271 ; \mathrm{S} 1<\mathrm{S} 4=0.195 \\
\mathrm{~S} 1<\mathrm{S} 5=0.406 ; & \mathrm{S} 2>\mathrm{S} 3=0.383 ; \mathrm{S} 2>\mathrm{S} 4=0.934 ; \\
\mathrm{S} 2>\mathrm{S} 5=0.514 ; \mathrm{S} 3<\mathrm{S} 4=0.393 ; \mathrm{S} 3<\mathrm{S} 5=0.845 & \mathrm{~S} 4>\mathrm{S} 5=0.532\end{array}$} \\
\hline & & S2 & 12 & 40.2 & 57.0 & \\
\hline & & S3 & 12 & 24.2 & 18.8 & \\
\hline & & S4 & 12 & 42.6 & 73.2 & \\
\hline & & S5 & 12 & 26.7 & 35.9 & \\
\hline
\end{tabular}

* significant differences were lower than 0.05

* diferenţele semnificative au fost mai mici de 0,05
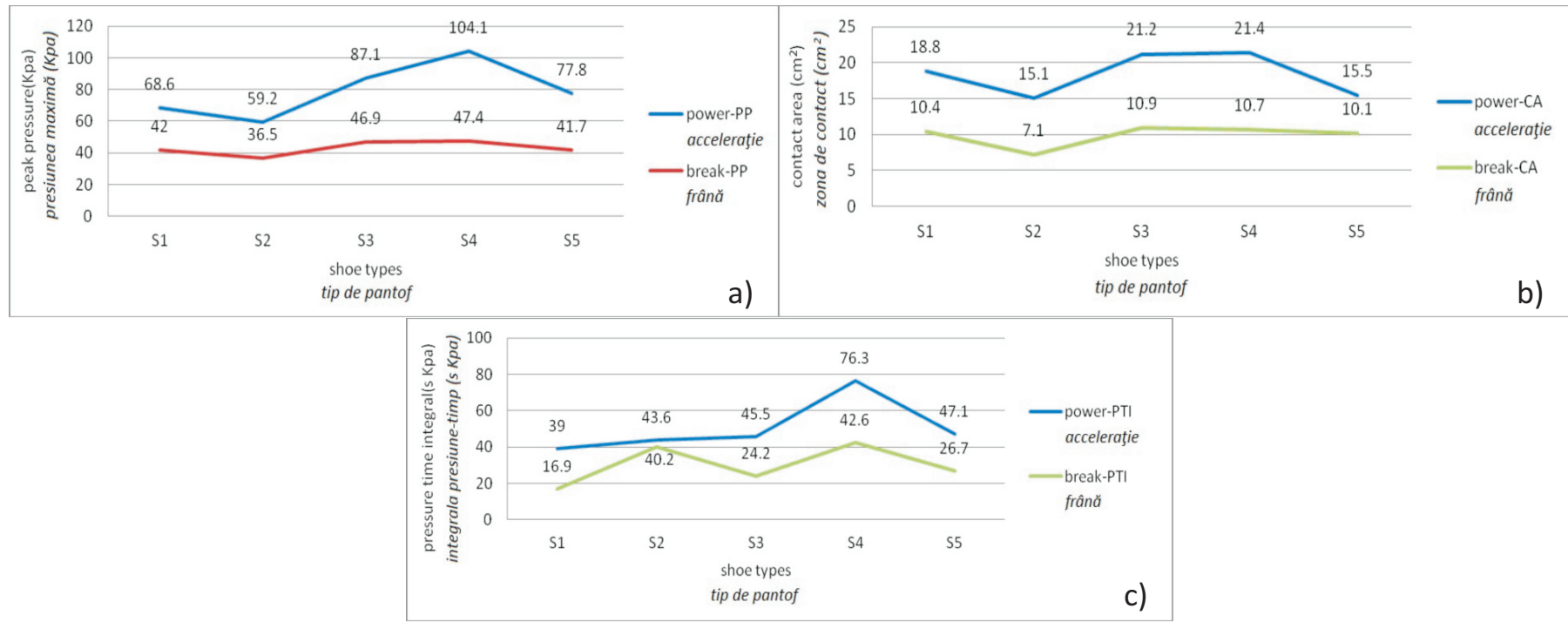

Figure 4. Contrast of plantar pressure distribution between action of power and break, where $A$ indicates the variable of $P P, B$ is $C A$, and $C$ is PTI

Figura 4. Comparaţie între distribuţia presiunii plantare la apăsarea pedalelor de acceleraţie şi frână, unde $A$ indică variabila PP, B este CA, iar C este PTI 


\section{Discussion}

In this study, the insole pressure distribution and ankle motion range whilst hitting the brake and power pedals were measured and the effects of heel height on the driving were systematically evaluated. Our results show that in terms of ankle motion, it increased with the relative heel height; in terms of insole pressure, only the CA was sensitive to the augment of relative heel height. Based on the significant differences, $30 \mathrm{~mm}$ heel height was a criterion; when the heel height exceeded $30 \mathrm{~mm}$, the ankle motion range and CA were significantly increased.

The existing research studies pointed out that, when wearing high-heeled shoes, pivot point was elevated dramatically [12], in which it actually added the range of ankle movement and forces in order to complete the break and power action. Influence of this mechanics was shown in the following aspects: (1) as the heel height increased, the angle of plantar flexion decreased and the ankle rotation was limited [13]; (2) the action of break when wearing high heel-heighted shoes was supported by the ankle rotation and the muscle group of tibialis posterior [14, 15], hence more time and more vector force would be consumed and generated in the whole process.

Given the absence of peer studies, our outcomes could not be compared with others'; however, on the one hand, we suggested that the heel height has no significant influence on the PP and the PTI. This outcome indicated that even when the same forces were output, the more energy would be consumed as the motion range increased as the heel height elevated. More energy consumption made fatigue become a risk factor in the driving. On the other hand, our results implied that significant differences were correlated with the absolute heel height, not with relative heel height, for instance, the performance of S4 and S5; while, we were not suggesting that the higher waterproof platforms, the safer driving condition. Because the thick platforms will lead to the extension of time for force transfer, and weaken the perception of spatial position when stepping on the pedal. Although quantitative outcomes were received in this study, there still are some limitations and should be comprehended with caution: (1) subjects might have their own driving habitudes and these might affect pressure distribution and ankle motion; (2)

\section{Discuţii}

În acest studiu s-au măsurat distribuţia presiunii la nivelul branţului şi amplitudinea articulară în timpul acţionării pedalelor de frână şi acceleraţie şi s-a evaluat sistematic influenţa înălţimii tocului asupra şofatului. Rezultatele noastre arată că în ceea ce priveşte amplitudinea articulară, aceasta a crescut odată cu înălţimea relativă a tocului; în ceea ce priveşte presiunea la nivelul branţului, doar CA a fost sensibilă la creşterea înălţimii relative a tocului. Pe baza diferenţelor semnificative, criteriul a fost tocul de $30 \mathrm{~mm}$ înălţime; atunci când înălţimea tocului a depăşit $30 \mathrm{~mm}$, amplitudinea articulară şi CA au crescut semnificativ.

Cercetările existente au subliniat că, în timpul purtării pantofilor cu toc înalt, punctul pivot a crescut dramatic [12], ducând la creşterea amplitudinii articulare şi a forţelor pentru a apăsa complet pedalele de frână şi acceleraţie. Influenţa acestui mecanism este relevată prin următoarele aspecte: (1) pe măsură ce a crescut înălţimea tocului, unghiul de flexie plantară a scăzut, iar rotaţia gleznei a fost limitată [13]; (2) apăsarea pedalei de frână purtând pantofi cu toc înalt a fost susţinută de rotaţia gleznei şi de muşchiul tibial posterior [14, 15], prin urmare, se consumă şi se generează mai mult timp şi mai multă forţă în întregul proces.

Având în vedere absența unor studii asemănătoare, rezultatele noastre nu au putut fi comparate cu cele ale altor cercetători. Totuşi, pe de o parte, am sugerat că înălţimea tocului nu are niciun efect semnificativ asupra PP şi PTI. Acest lucru a indicat că, deşi au rezultat aceleaşi forţe, se consumă mai multă energie pe măsură ce creşte amplitudinea articulară odată cu creşterea înălţimii tocului. Un consum mai mare de energie înseamnă că oboseala devine un factor de risc în timpul şofatului. Pe de altă parte, rezultatele noastre au indicat că diferenţele semnificative au fost corelate cu înălţimea absolută a tocului, nu cu cea relativă, de exemplu, performanţa pantofilor S4 şi S5. Nu am sugerat că utilizarea unor platforme înalte rezistente la apă contribuie la un şofat mai sigur, deoarece platformele groase duc la prelungirea timpului de transfer al forţei şi slăbesc percepţia poziţiei spaţiale la apăsarea pedalei. Deşi s-au obţinut rezultate cantitative în acest studiu, există încă unele limitări şi trebuie înţelese cu precauţie: (1) subiecţii au propriile lor obiceiuri când vine vorba de şofat şi acestea ar putea afecta modul de distribuţie a presiunii şi mişcarea gleznei; (2) tipurile şi materialele de încălţăminte din acest studiu nu au fost luate în 
styles and materials of footwear in this study were not considered and this might influence the comfort perception whilst driving. Based on the finding of this study, future work will focus on the designing of a safety driving footwear for female drivers.

\section{CONCLUSIONS}

Overall, based on the above finding, we concluded that the safe heel-height of driving shoe should not exceed $30 \mathrm{~mm}$.

\section{Acknowledgements}

The author was pleased to thank the financial support from: China Postdoctoral Science Foundation (2015M571896), National Science and technology support program (2014BAE02B02) and Funding of Sichuan University (2014SCU11029). considerare şi acest lucru poate afecta percepţia confortului în timpul şofatului. Pe baza constatărilor acestui studiu, cercetările viitoare se vor concentra pe proiectarea unui articol de încălţăminte pentru şofat în siguranţă destinat conducătorilor auto de sex feminin.

\section{CONCLUZII}

În general, pe baza constatărilor de mai sus, am ajuns la concluzia că, pentru siguranţă, înălţimea tocului încălţămintei utilizate la şofat nu trebuie să depăşească $30 \mathrm{~mm}$.

\section{Mulţumiri}

Autorul mulţumeşte pentru sprijin financiar Fundaţiei Ştiinţifice pentru Studii Postdoctorale din China (2015M571896), Programului Naţional de Sprijin pentru Ştiinţă şi Tehnologie (2014BAE02B02) şi Universităţii Sichuan (2014SCU11029).

\section{REFERENCES}

1. WHO, Global Status Report on Road Safety 2013: Supporting a decade of action, Geneva, WHO, 2013.

2. Zhang, W., Huang, Y.H., Roetting, M., Wang, Y., Wei, H., Accid Anal Prev, 2006, 38, 22-7.

3. Yang, J., Du, F., Qu, W., Gong, Z., Sun, X., Traffic Inj Prev, 2013, 14, 565-71.

4. Leung, P.S., The prevalence of domestic violence among the female Chinese population in the accident and emergency department, Postgraduate thesis, The University of Hong Kong, 2002.

5. Popkin, C.L., Rudisill, L.C., Waller, C.F., Geissinger, S.B., Accid Anal Prev, 1988, 20, 219-225.

6. Guang, F., Auto and Safety, 2012, 5.

7. Junliang, F., Weihua, W., Zibai, X., Auto and Safety, 2005, 7, 53-53.

8. Jin, H.Q., Araki, S., Wu, X.K., Zhang, Y.W., Yokoyama, K., Int J Epidemiol, 1991, 20, 230-3.

9. Vasilescu, A.M., Petrescu, C., Movila, C., Revista de Pielarie Incaltaminte (Leather and Footwear Journal), 2011, 11, 2, 149-162.

10. Vasilescu, A.M., Petcu, D., Micu, A.C., Revista de Pielarie Incaltaminte (Leather and Footwear Journal), 2009, 9, 3, 155-174.

11. Vasilescu, A.M., Berijan, G., Jidiuc, B., Petcu, D., Revista de Pielarie Incaltaminte (Leather and Footwear Journal), 2008, 8, 3, 35-43.

12. Stefanyshyn, D.J. et al., J Appl Biomech, 2000, 16, 3, 309-319.

13. Kouchi, M., Tsutsumi, E., Anthropol Sci, 2000, 108, 4, 331-343.

14. Speksnijder, C. et al., The Foot, 2005, 15, 1, 17-21.

15. Cho, W.H., Choi, H.K., Advances in Fracture and Strength, 1-4, Trans Tech Publications, Y.J. Kim and H.D. Bae, Editors, 2005, 2303-2307.

Article received/Data primirii articolului: 14.05.2015

Accepted/Acceptat la data: 23.07.2015 